\title{
Interpolated Observations and Historical Observational Records in Ptolemy's Astronomy
}

\author{
Alexander Jones
}

This paper considers two subsets of the dated observation reports in Ptolemy's Almagest: reports that describe a phenomenon or celestial configuration supposedly in effect at the stated date, but that were derived from an underlying series of actual observations made over a span of time ("interpolated observations"), and reports of observations made more than a century before Ptolemy's time ("historical observations"). Where these subsets intersected, Ptolemy was confronted with interesting problems arising from transitions in observational practice and losses of information through the processes of transmission from the original observers.

2

Interpolated Observations and Their Place in Ptolemy's Astronomical Deductions

Ptolemy's Mathematical Composition, better known as the Almagest, presents

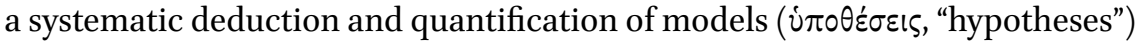
based on uniform circular motions to explain the motions and phenomena of the heavenly bodies, based on and in agreement with empirical data. The book's methodology is extremely complex and sophisticated, and the empirical or observational elements enter into its arguments in diverse ways. In the broadest terms, Ptolemy summarizes what he regards as a satisfactory deduction of a model in Almagest 9.2 as involving three stages:

(1) demonstration of the periodicities and sizes of the various geometrical components of the model from manifest and agreed-upon "phenomena";

(2) demonstration of how these elements are to be combined in the model; and

(3) demonstration that the model accounts for practically all the phenomena (not just the phenomena used in the first stage). 
In practice he does not go through these stages in a straightforward linear order. For example his treatment of the planet Mars has the following structure:

(1a) assumption of periodicities obtained in the first instance from the research of a predecessor (Hipparchos) but subject to confirmation and correction that will come at a later stage (Almagest 9.3);

(2a) assumption of an unquantified model structure (epicycle and eccenter model with equant and sidereally precessing apsidal line), some elements of which - the epicycle to account for the synodic anomaly and the eccenter for the zodiacal anomaly — are justified at this stage on the basis of generalized phenomena whereas the justification of the others is deferred (Almagest 9.5-6);

(2b) demonstration of the precession of the apsidal line of Mercury, based on analysis of dated observations, and extended to the other four planets on the basis of an unspecific assertion that "the phenomena of the other planets individually fit" (Almagest 9.7);

(2c) demonstration of the equant and its location from generalized phenomena (Almagest 10.6);

(1b) quantification of the eccentricity, epicycle radius, corrected periodicities, and epoch from dated observations (Almagest 10.7-10);

(2d) representation of the quantified model in predictive form using trigonometry or a table of anomaly, allowing computation of the planet's longitude for a given date (Almagest 11.9-12);

(3a) demonstration of retrogradations predicted by the quantified model ( $\mathrm{Al}$ magest 12.4 and $12.7-8$ );

(2e) demonstration of the unquantified latitudinal inclinations of the model's geometrical components from generalized phenomena (Almagest 13.1-2);

(1c) quantification of the inclinations from undated observations (Almagest 13.3);

(2f) representation of the quantified model in predictive form using a table of latitude, allowing computation of the planet's latitude for a given date (Almagest 13.4-6); and

(3b) demonstration of first and last visibilities predicted by the quantified model (Almagest $13 \cdot 7$ and 13.9-10).

Thus we encounter explicit empirical input in all the stages except those that merely work out the predicted behavior of the model as it has been deduced so far. (Stages $3 \mathrm{a}$ and $3 \mathrm{~b}$ implicitly invite comparison with the observable behavior of the planet, but Ptolemy does not adduce any empirical confirmation of his results in so many words.) The empirical evidence comes in several varieties: 
generalized phenomena, i.e. empirical claims such as "the time from least speed to mean (of a planet in its synodic cycle) is always greater than the time from mean speed to greatest".

Almagest 9.5, $\mathrm{H} 2.251$

dateless measurements, such as "at the oppositions near the apogee (Mars) is $41 / 3^{\circ}$ to the north of the ecliptic".

Almagest 13.3, $\mathrm{H} 2.539$

dated records of immediate observations, such as "in the second year of Antoninus, Epiphi 15/16 in the Egyptian calendar, 3 equinoctial hours before midnight, ... Mars was seen to have a longitude of Sagittarius 1 $3 / 5^{\circ}$.

Almagest 10.8, H2.347

dated statements of events that depend on interpolating between observations made before and after, such as "(Mars was in opposition to the mean Sun) in the fifteenth year of Hadrian, Tybi 26/27 in the Egyptian calendar, 1 equinoctial hour after midnight, at about Gemini $21^{\circ}$ ".

Almagest 10.7, H2.322

The distinction between an immediate observation and an interpolated observation is this: in an immediate observation, either the time for observing a desired configuration of the heavenly bodies could be chosen by the observer, or the timing of the configuration was directly manifest; in an interpolated observation, either the direct observations by autopsy could only be made at certain times that would not normally coincide with the desired configuration, or the timing of the configuration was not straightforwardly observable, or the configuration can be recognized only in relation to observations at other times, or the configuration could not in any case be directly observed.

Among the dated observations cited in the Almagest, the following types are immediate:

1 Translations of brief passages in the Almagest are adapted from Toomer (1984), with some minor stylistic modifications; parentheses enclose supplements and glosses to Ptolemy's wording. Page references in the form "H2.322" are to the volume and page number in Heiberg's edition, which are also provided in the margins of Toomer's translation. For a general discussion of the empiricism of the Almagest and the respective roles of generalized phenomena and specific observations see Swerdlow (2004). 
elongations of the Moon from the Sun (date and time may be chosen at the observer's convenience so long as both luminaries are visible)

positions of Moon or a planet in relation to fixed stars (date and time may be chosen at the observer's convenience)

lunar eclipses (times of beginning and end of obscuration and totality are directly observable $)^{2}$

whereas the following types are interpolated:

solstices (direct observations made only at noon, requires comparison with observations on other dates)

equinoxes (direct observations made only at noon)

oppositions of a superior planet with mean Sun (timing of event not straightforwardly observable, frequently occurring at time when direct observation is not possible)

greatest elongations of an inferior planet from the mean Sun (requires comparison with observations on other dates, direct observations made only in first or last part of night)

The basic principle of an interpolated observation is that the assertion that such-and-such a configuration or phenomenon occurred at such-and-such a date and time is an inference based on a series of direct observations made over span of time, say, at the same time on several successive days, which are not individually reported. In some kinds of observation, one is trying to determine the date when some extreme value is reached in the middle of an interval during which the value is changing very slowly. Ptolemy's extremal observations include solstices and greatest elongations; dated observations of planets' stations, which Ptolemy does not cite in the Almagest, would also be extremal. In other kinds, one is looking for the date when a more rapidly and monotonically changing quantity crosses a threshold value. In the Almagest,

2 Since Ptolemy generally requires the time of mid-eclipse, these observations are in a trivial sense interpolated, slightly less trivial in cases where the time of beginning or end was not observable or not recorded. 
observations of equinoxes and planetary oppositions are of this type, as would be observations of planetary conjunctions if Ptolemy had made use of them. When applying interpolated observations for theoretical work, one always requires the date of the desired configuration; in the case of the planetary observations one also needs the planet's longitude on that date.

The fact that Ptolemy never gives us the series of actual observations from which the interpolated observations were obtained limits what we can know about the methods of interpolation that he or his sources used. Nevertheless one can at least distinguish some basic approaches. In some cases, it appears that someone has simply selected from the series of direct observations the one that most closely corresponds to the desired configuration. In others, the date evidently falls between direct observations, so that it and the associated longitude (if the configuration involves a planet) must have been obtained by some kind of computation.

A third category arises with respect to greatest elongations of Mercury and Venus. Ptolemy uses these as surrogates for observations of the invisible points where a tangent line from the observer touches the planet's epicycle. If he requires such an observation in a situation where no greatest elongation has taken place within the range of available observations, one solution available to him is to interpolate a fictitious observation between actual greatest elongations observed in nearby situations, simulating what would have been observed if the planet had been at a point near the point of tangency instead of some other point on its epicycle. These constitute a kind of second-order interpolated observation.

\section{Historical Observations and Their Place in Ptolemy's Deductions}

The dated observations reported in the Almagest, other than those that Ptolemy identifies as his own, were made by many disparate individuals and groups: ${ }^{3}$

Babylonians: ten lunar eclipses ( $721-382 \mathrm{BC})$, three observations of positions of planets (Mercury and Saturn) relative to stars $\left(245^{-229}\right.$ BC).

Those around (oi $\pi \varepsilon p i)$ Meton and Euktemon: $:^{4}$ summer solstice (432 BC).

3 The reports can be located by means of Toomer's index, by the observer, the heavenly body, or the kind of event.

4 In Ptolemy's usage, the expression "those around" seems to indicate a degree of vagueness or uncertainty adhering to observations ascribed to chronologically remote individuals. 
Timocharis: four observations of positions of Moon relative to stars (295$283 \mathrm{BC}$ ), two observations of Venus relative to stars (272 BC), and some lunar eclipse observations (dates not given in the Almagest).

Those around Aristarchos: summer solstice $(28 \mathrm{o} \mathrm{BC})$.

Unnamed observers using the calendar of Dionysios, in Alexandria (?): 5 ten observations (henceforth "Dionysian observations") of positions of the planets Mercury, Mars, and Jupiter relative to stars (272-241 BC).

Archimedes: solstice observations (dates not given in the Almagest).

Unnamed observers in Alexandria: four lunar eclipses (201-174BC), vernal equinox (146 вC).

Hipparchos: autumnal and vernal equinoxes (162-128 вC), summer solstice $(135 \mathrm{BC})$, three lunar eclipses $(146-135 \mathrm{BC})$, three lunar elongations (128-127 BC).

Agrippa: observation of position of Moon relative to stars (AD 92).

Menelaos of Alexandria: two observations of positions of Moon relative to stars (AD 98).

Theon the Mathematician: four observations of positions of planets (Venus and Mercury) relative to stars (AD 127-132)

A significant chronological divide is apparent between the observations up to Hipparchos's time, which for convenience we will call "historical," and those dating from the late first and second centuries of our era, which we will call "recent." As a rule, Ptolemy bases his deductions and quantifications of models on his own observations or other recent observations (Agrippa, Menelaos, Theon) except when he wishes to demonstrate a long-term periodicity or rate of motion. For such demonstrations, he generally employs historical observations dating from the remotest period from which he possessed reports of the right kind and of suitable quality. Exceptions to this rule include several histor-

5 For the evidence situating these observers in Alexandria, or at least in Ptolemaic Egypt, see Jones (2006:257). 
ical lunar eclipse reports that satisfy special constraints that perhaps were not satisfied by any recent report available to Ptolemy, and observations by Hipparchos of lunar elongations which Ptolemy adduces in his deduction of the eccenter-and-epicycle model for the Moon (Almagest 5.3 and 5.5), ostensibly to back up his claim that he discovered the necessity of this model through studying Hipparchos's observations. In the context of discussing aspects of Hipparchos's researches Ptolemy also cites various historical observations used by Hipparchos but that play no role in Ptolemy's own deductions.

With the probable exceptions of the summer solstices associated with Meton and Euktemon and with Aristarchos, which both appear to have been inaugural dates for calendrical cycles, ${ }^{6}$ all the historical and recent observations in the Almagest were probably selections from larger sets compiled by the various individuals or groups. How were these sets presented by their authors, and did Ptolemy have access to them in their original context or only through intermediaries? There existed essentially two modes of presentation for observation reports: corpora collecting reports as a resource for reference or research, and theoretical or didactic texts (such as the Almagest itself) in which selected reports were embedded. We can identify three of the corpora from which some of Ptolemy's reports derived, and interestingly, they illustrate diverse paths by which reports passed from the original observers to their ultimate user, Ptolemy:

Theon the Mathematician: ${ }^{7}$ Ptolemy speaks of "an observation recorded among those given to us by the mathematician Theon" ( $\dot{\varepsilon} \nu$... $\tau \alpha \hat{\imath} \varsigma \pi \alpha \rho \dot{\alpha}$

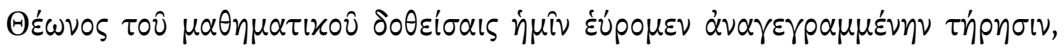
Almagest 10.1, H2.296), and again another "among the observations we

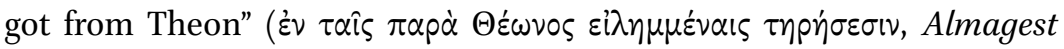
9.9, H2.275). This was evidently a corpus compiled by the observer and communicated personally to Ptolemy.

Babylonians: Ptolemy's Babylonian eclipse reports probably, and his three Babylonian planetary observations almost certainly, derive from cunei-

6 The Meton-Euktemon solstice date is cited as an inaugural date for a 19-year calendrical cycle by Diodoros 12.36 and IMilet inv. 84, for which see Diels \& Rehm (1904:93-97), Dessau (1904), Lehoux (2005), and Jones (forthcoming). The Aristarchos date is exactly eight 19-year cycles later, as noted by Dessau.

7 Theon the Mathematician is not known from any source independent of the Almagest; he is not to be identified with the contemporary Platonist philosopher Theon of Smyrna, whose grasp of planetary theory was inaccurate and out of date by the standards of Ptolemy's time. 
form tablets in the astronomical archive of Babylon, substantial portions of which are extant in the British Museum and other modern collections. The source documents would have been corpora, either the Astronomical Diaries, which were the original form of record in which observations of a wide range of astronomical phenomena were organized chronologically over spans of several months, or various types of excerpt tablets that listed observations of a single kind in chronological order over longer spans. The first presentations of these reports in Greek are likely also to have been corpora. Several of the Babylonian eclipses in the Almagest are known to have been cited by Hipparchos in works to which Ptolemy had access; it is likely, though not certain, that Ptolemy was also able to draw on corpora containing Greek versions of eclipse and planetary observations.

Hipparchos: Ptolemy writes (Almagest 9.2, 2.210) that Hipparchos's only contribution to planetary theory, to his knowledge, was "to make a compilation of the planetary observations arranged in a more useful way, and to show by means of these that the phenomena were not in agreement with the hypotheses of the astronomers of that time." This might have been two separate books or a single one in two parts, but the "compilation" was clearly a corpus that collected planetary observation reports by earlier astronomers as well, perhaps, as by Hipparchos himself. That Ptolemy had access to it, or to some derivative of it, is confirmed by one of the Dionysian observations cited in Almagest 9.7, where he gives Hipparchos's reduction of the report (Mercury "was a little more than $3^{\circ}$ in advance of Spica, according to Hipparchos's reckoning", H2.267) rather than the original wording.

Let us now turn to the evidence for theoretical treatises by Ptolemy's predecessors that contained reports subsequently cited by Ptolemy:

Hipparchos's On the Displacement of the Solstitial and Equinoctial Points, according to Ptolemy's discussion of it in Almagest 3.1, cited lunar eclipse observations by Timocharis, solstice observations by Archimedes, Hipparchos's own observations of solstices and equinoxes and of two lunar eclipses, and at least one observation of an equinox made by a contemporary at Alexandria (likely obtained by personal communication). Among these, Ptolemy reused for his own theoretical deductions one of Hipparchos's vernal equinoxes and one of his autumnal equinoxes. 
Almagest 3.1 (H1.206-207) cites Hipparchos's On the Length of the Year as having made use of the Aristarchos solstice and of his own summer solstice observation of $135 \mathrm{BC}$. It is likely that the Meton-Euktemon solstice was also discussed in this work (as implied by a quotation from it in Almagest 7.2, $\mathrm{H} 2.15^{-16)} .{ }^{8}$ Of these, Ptolemy reused the Meton-Euktemon solstice.

Almagest 4.11 discusses what may have been either one treatise or two by Hipparchos on the measurement of the Moon's anomaly, in which he cited three Babylonian eclipse observations from $383-382$ BC and three Alexandrian lunar eclipse observations from 201-200 BC. Ptolemy does not use these observations in his own deductions, though he appeals to their agreement with his own solar and lunar theory as a verification of its validity.

Almagest 6.9 summarizes part of the argument of a work of Hipparchos's (possibly the book On the Moon's Monthly Motion in Latitude mentioned

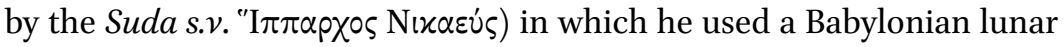
eclipse observation from $720 \mathrm{BC}$ together with one of his own from $141 \mathrm{BC}$ to establish the Moon's periodicity in latitude. Both observations were used by Ptolemy for different purposes (Almagest 4.6, 4.9, and 6.5).

Almagest 4.9, in citing a Babylonian eclipse observation from 502 BC, adds

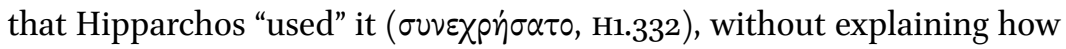
or in what context.

In Almagest 11.3 (H2.386), Ptolemy makes use of a Dionysian observation of Jupiter from $241 \mathrm{BC}$ that he very probably found in an early and century AD theoretical treatise of which a fragment is extant in the papyrus POxy astron. $4133 .{ }^{9}$ This treatise may have been by Menelaos of Alexandria. The part preserved in the papyrus cites a different observation of Jupiter made in $241 \mathrm{BC}$, just four months after the one in the Almagest, as well as one made by the author in AD 104.

Beyond this evidence we can only speculate. For the recent observations other than those of Theon, it seems at least plausible that Ptolemy's sources were

8 See Toomer (1974:328 note 53).

9 Jones (1999). 


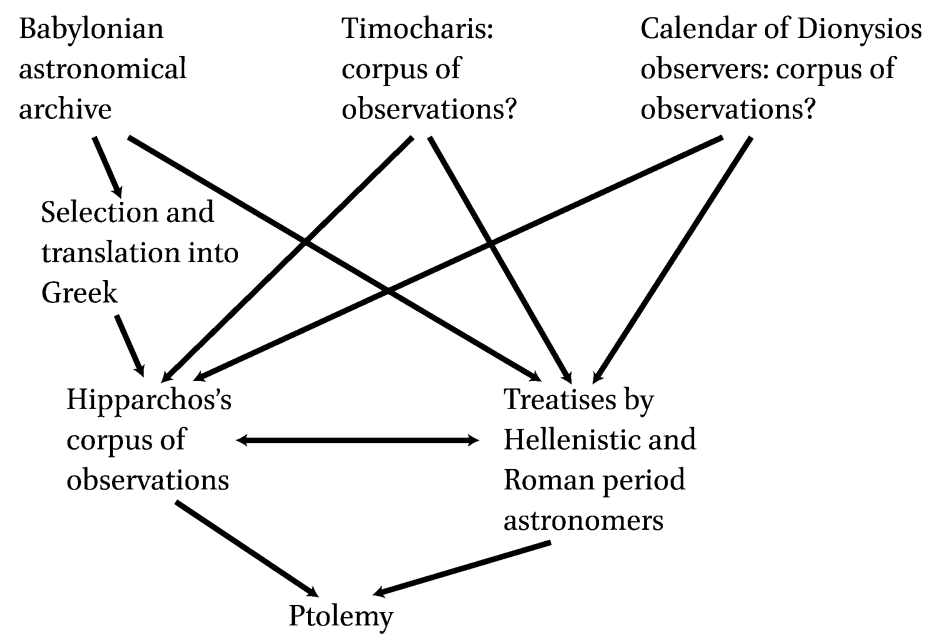

FIGURE 10.1 A conjectural scheme for Ptolemy's reception of historical planetary observations

treatises by Agrippa (concerning whom we know nothing besides the single report in Almagest 7.3, H2.27) and Menelaos. I would guess that the original presentation of the historical observations of Timocharis and the unknown astronomers who employed the calendar of Dionysios took the form of corpora, but we have no evidence that Ptolemy had direct access to these corpora or indeed to any text from before Hipparchos's time that contained observation reports. It is worth noting that Ptolemy seems to have had at least two indirect channels transmitting Dionysian observations, namely Hipparchos's corpus and the early second century AD treatise on Jupiter. Fig. 10.1 shows a schematic reconstruction of the routes by which the historical observations of the planets could have reached Ptolemy without presumption that he consulted sources older than Hipparchos.

While Ptolemy appears to have regarded even his historical eclipse reports, including even the oldest ones from the eighth century BC, as satisfactory for his analytic purposes, he is more critical of the pre-Hipparchian solar and planetary observations. He writes that the summer solstice reports associated with Meton and Euktemon (432 BC) and with Aristarchos (280 BC) "were conducted rather crudely, as Hipparchos too seems to think" (Almagest 3.1, H1.203), though he nevertheless makes use of the earlier of them "because of its antiquity" (H1.205). As for the early observations of the planets, he has several complaints (Almagest 9.2, H2.209-210): 
they do not extend as far back in time as one would like;

the "more continuous series of observations" ( $\sigma \nu v \varepsilon \chi \varepsilon \dot{\varepsilon} \sigma \varepsilon p \alpha)$ consist of first and last visibilities and stations, which cannot be observed accurately and unambiguously; and

observations of the locations of planets relative to fixed stars, unless the distances are small, are hard to calculate and hit-or-miss ( $\sigma \tau \sigma \chi \alpha \sigma \tau i x \eta \dot{v} \nu)$ a judgment that seems to presume that such observations were carried out without instruments, which may well have been true.

In practice Ptolemy makes no use of observations of stations and visibility phenomena. His historical observations of positions of planets relative to stars are spread over the interval $272-229 \mathrm{BC}$, from which we can infer that his sources provided few if any satisfactory observations of this kind from before the early third century вс. ${ }^{10}$ The ones that he selects are by no means limited to situations where the planet is very close to a star; in one instance, a Dionysian observation of Mercury from $257 \mathrm{BC}$ (Almagest 9.7, H2.265), the reported distance of the planet from the nearer of the two stars mentioned is more than $9^{\circ}$ according to Ptolemy's reckoning.

At this point we can turn to consideration of those among Ptolemy's historical observations that are also interpolated observations. These turn out to be of just two kinds: solar observations of solstices and equinoxes, and observations of an inferior planet (Mercury) at greatest elongation from the mean Sun. In both cases, the materials that Ptolemy had to work with set severe limits on the usefulness of the observations, though for different reasons.

As we noted above, all the historical solstice and equinox observations mentioned in the Almagest were cited in Hipparchos's On the Displacement of the Solstitial and Equinoctial Points, and it is possible that this work was effectively Ptolemy's sole source for them. Dated reports of solstice observations from before Hipparchos's time seem in fact to have been quite rare and dated reports of pre-Hipparchian equinoxes practically nonexistent. The $432 \mathrm{BC}$ date associated with Meton and Euktemon was in fact famous enough in antiquity to be

10 On these observations see Jones (2006). 
cited by a public inscription at Miletos (IMilet inv. 84, dating after $109 \mathrm{BC}$ ), as well as by the first century вС historian Diodoros, ${ }^{11}$ though Diodoros does not identify the date as a summer solstice and neither source assigns a time of day to it as Ptolemy does. Without Ptolemy we would not even know of the existence of the Aristarchos solstice report of $28 \mathrm{o} \mathrm{BC}$ or the series of solstices of unknown date observed by Archimedes.

The crucial question with respect to the solstices of 432 and $280 \mathrm{BC}$ is why

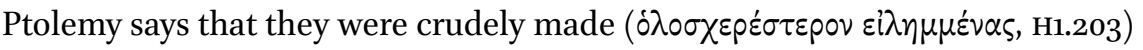

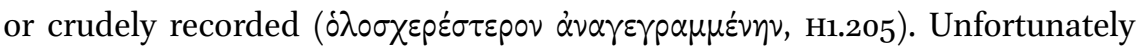
Ptolemy does not provide us with much of a basis for deciding what he (or Hipparchos before him) would have regarded as a crude or an accurate solstice report. The specific solstices mentioned in the Almagest, all of them in 3.1 and all of them summer solstices, are the following (giving the proleptic Julian calendar equivalents of Ptolemy's Egyptian calendar dates):

432 BC June 27, at daybreak ( $\pi$ p wís), observed "crudely" by "those around Meton and Euktemon" (H1.205-206)

28 о BC, date and time not given, observed "crudely" by "those around Aristarchos" (H1.203 and 206-207)

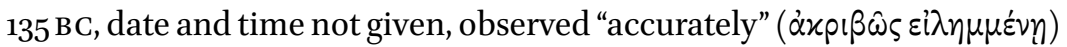
by Hipparchos (H1.207)

AD 140 June 25, about 2 hours after midnight, "computed securely" ( $\alpha \sigma \varphi \alpha-$

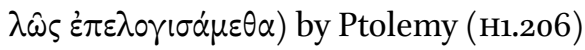

The only information provided in the two more detailed reports is the date and time. Ptolemy gives only the years for the 280 and 135 BC solstices, but he quotes Hipparchos as saying that the time interval between them was 52960 1/2 days. Moreover an indirect argument from a year length attested in a Babylonian astronomical tablet, BM 55555, indicates with high plausibility that Hipparchos calculated the time interval between the $43^{2}$ and 135 BC solstices as 108478 days exactly. ${ }^{12}$ It seems likely, therefore, that Hipparchos was treating the times as if

11 See note 6 above.

12 Rawlins (1990:49-51); Jones (2005:23-24). For the tablet (also known as ACT 210) see Ossendrijver (2012:496-500). If Hipparchos's observed time for the solstice of 135 BC was 108478 days later than the assumed time of the Meton-Euktemon solstice, it would have been on June 26 at daybreak. The true moment of solstice was on June 26 at about 8 AM. 
they were precise to the quarter day: effectively noon, 6 PM, midnight, and 6 AM. This is concordant with his statement, quoted by Ptolemy (Almagest 3.1, H1.194195), that "both I and Archimedes may have committed errors of up to a quarter of a day in our observations and calculations" of the times of solstices, though strictly speaking this is a statement about accuracy, not precision. The reports of Hipparchos's equinox observations that are cited in Almagest 3.1 (H1.195-196) all have such quarter-day times.

By chance a report of another summer solstice observed by Hipparchos has recently come to light in a source independent of Ptolemy, a fragment of an instructional text on astronomical calculations in the papyrus PFouad 267A, recto lines $10-12 . .^{13}$ The date of the solstice is given as $158 \mathrm{BC}$, June 26 , at a seasonal hour of the day whose number the papyrus's scribe unfortunately omitted to write. A combination of information in the papyrus allows us to estimate the time of the solstice in question according to the solar tables that the text's author was using as having been about an hour after sunset. The tables were allegedly framed in agreement with Hipparchos's observations, ${ }^{14}$ of which the solstice of $158 \mathrm{BC}$ is the only one specifically mentioned, but we cannot tell how precise the fit really was. ${ }^{15}$ All we can say, therefore, is that in this papyrus's source a Hipparchian solstice observation was reported to an ostensible precision of hours, but it cannot be excluded that this was merely someone's way of expressing a time that Hipparchos recorded as precise to the quarter day.

Quarter-day precision would be a reasonable outcome of a simple method of interpolation applied to observations made at one-day intervals around the expected dates of solstices or equinoxes, which for Hipparchos would most probably be observations of the Sun's altitude at noon made by means of a meridian instrument similar to the ones that Ptolemy describes in Almagest 1.12. Because the daily change in solar declination approaches zero at the solstices, Hipparchos would likely have estimated the moment of solstice as halfway between two dates of observed equal declination sufficiently before and after the solstice so the daily change is perceptible. Supposing two such observations were found, the solstice would have been estimated as occurring at noon if the interval between the pair was an even number of days or

13 Fournet \& Tihon (2014:22-23 and 28-29).

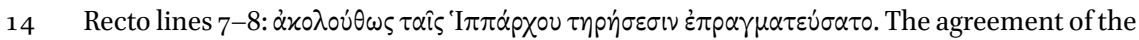
model with the report of Hipparchos's observation of the autumnal equinox in $159 \mathrm{BC}$, which is transmitted in Almagest 3.1 but not in the papyrus, is almost perfect.

15 The true moment of solstice was on June 26 at about 6 PM. 
midnight if the interval was an odd number of days. If, however, the observation preceding the solstice was not found to have an exact counterpart after the solstice, but instead the declinations of two consecutive post-solstice observations appeared to flank that of the pre-solstice observation, the interpolated time of solstice would be between midnight and noon or between noon and midnight, and $6 \mathrm{AM}$ or $6 \mathrm{PM}$ would be a reasonable estimate. Refining the time to the higher precision of single hours, as Ptolemy appears to do for his own solstice observation, would have required an instrument enabling measurements of declinations on the order of single minutes.

In his On the Displacement of the Solstitial and Equinoctial Points Hipparchos probably did not report the individual noon observations from which he derived his interpolated solstice times, but since the book is known to have contained many such solstice times, Ptolemy would have known the precision with which they were expressed and likely he would have at least guessed how Hipparchos obtained this precision. But what would Hipparchos or Ptolemy have known about the older solstice reports from 432 and $280 \mathrm{BC}$, which were probably both transmitted as one-off dates rather than selected from larger sets? I think a plausible explanation of Ptolemy's assessing them as crude is that they were originally reported without specification of time of day, as we find the $432 \mathrm{BC}$ date recorded in Diodoros and in the Miletos inscription. Hipparchos would therefore have received them as observations having a precision of one day, and conjectured that each report represented the observation from among a series of consecutive observations for which the solar declination was greatest. He would have assigned the 432 BC observation to "morning," guessing that Meton and Euktemon were making observations of the Sun's rising point on the horizon (a reasonable assumption for this period), and the $28 \mathrm{o} \mathrm{BC}$ observation to noon in the belief that Aristarchos observed the Sun's meridian altitude.

If the foregoing reconstruction is correct, it casts an interesting sidelight on Hipparchos's attempts to measure the length of the mean tropical year ("mean" because, unlike Ptolemy, he suspected that this parameter was not a constant) and on Ptolemy's ostensible confirmations of the value $3651 / 4^{-}$ 1/300 days that Hipparchos apparently adopted. This value was obtained from, or at least consistent with, the interval between the 280 and 135 BC solstices, whereas the value obtainable from comparing the 432 and 135 BC solstices, if this is what вм 55555 gives us, was significantly less, closer to $3651 / 4-1 / 240$ days. Why did Hipparchos prefer the value obtained from an interval that was only about half as long as the longest interval available to him? The precision of the times was not the decisive issue, if we have correctly interpreted the charge of "crudeness" that Ptolemy makes against both. Hipparchos probably had greater 
doubt about the accuracy of the $432 \mathrm{BC}$ observation, and perhaps also was less confident about the synchronization of its reported Athenian calendar date with the chronological systems (the Egyptian and Kallippic calendars) that he used for his own observations.

For Ptolemy the time scale of available solstice observations was about double that available to Hipparchos, if for the sake of argument we treat his own solstice observations as genuine. He would have known that it was methodologically preferable to compare observations from his own time with Hipparchos's observations (or possibly still better, Archimedes's observations), rather than the earliest ones, both because their claimed precision more than compensated for the shorter time span, and because they were transmitted to him in the context of larger data sets that allowed a better assessment of their accuracy. That he chose to demonstrate the length of the tropical year using the least suitable of the historical solstice observations available to him is symptomatic of the highly dialectical character of Almagest 3.1. ${ }^{16}$

\section{$5 \quad$ Greatest Elongations: The Case of Recent Observations}

Almost the entire process of deduction and quantification of the models for Mercury and Venus in Almagest 9 and 10 is carried out using observations of a single type, greatest elongations of the planet; only the final stage of correction of the periodicities for each planet is based on analysis of a pair of observations of the planet's position at arbitrary dates, one of which is a historical observation. Ptolemy uses no historical observations of greatest elongations of Venus in the Almagest, but he provides two determinations of Mercury's apsidal line, one based on a set of recent greatest elongations, the other on a historical set. His purpose is to demonstrate that the apsidal line has shifted eastward in his tropical frame of reference by a rate of $1^{\circ}$ per century, which is identical to his rate for precession so that Mercury's apsidal line is sidereally fixed. Although he declares that this is true of the apsidal lines of all five planets, Mercury is the only one that gets a demonstration of this kind.

The configuration we are calling, for short, a "greatest elongation" is properly speaking a moment of greatest elongation from the mean Sun, that is, a moment when the difference between the true longitude of the planet and the mean longitude of the Sun attains a local maximum. The theoretical interpre-

16 See Jones (2005:18-27) for a broader discussion of this chapter. 


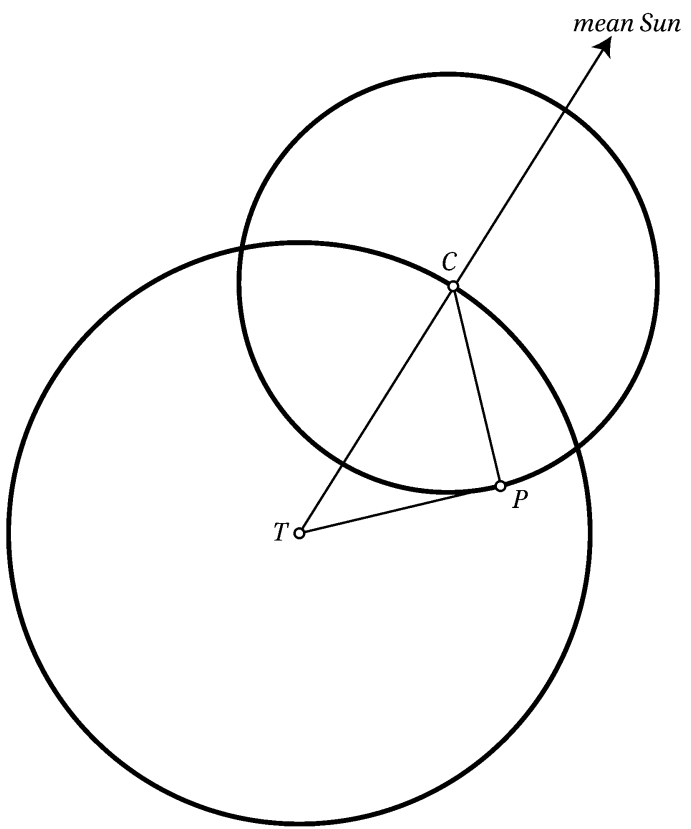

FIGURE 10.2 A simple epicyclic model for an inferior planet, shown approximately to scale for Venus

tation of this configuration depends on what kind of model is assumed. If it is a simple epicyclic model for an inferior planet (Fig. 10.2, drawn to scale for Venus), such that the planet $P$ revolves uniformly around its epicycle while the epicycle's center $c$ revolves uniformly, in alignment with the mean Sun, along a deferent circle concentric with the Earth $T$, greatest elongation occurs when the planet is exactly at either of the two points where a tangent from the center of the Earth touches the epicycle. According to the simple model the arc of greatest elongation is a constant (positive if the planet is currently visible in the evening, negative if visible in the morning), dependent only on the ratio of radii of the epicycle and deferent, and the time intervals between successive greatest elongations of the same sign equal to the synodic period of the planet, which is also constant in this model.

In Ptolemy's planetary models with eccentric deferent and equant, the interpretation of a greatest elongation is more complicated (Fig. 10.3, drawn to scale for Venus). Here it is the radial line from the equant $E$ to the epicycle's center $c$ that is aligned with the mean Sun, whereas the planet's true longitude is the direction of the radial line from the Earth's center $T$ to the planet $P_{1}$. When the angle between these radii is maximum, the planet is not, in general, at the exact 


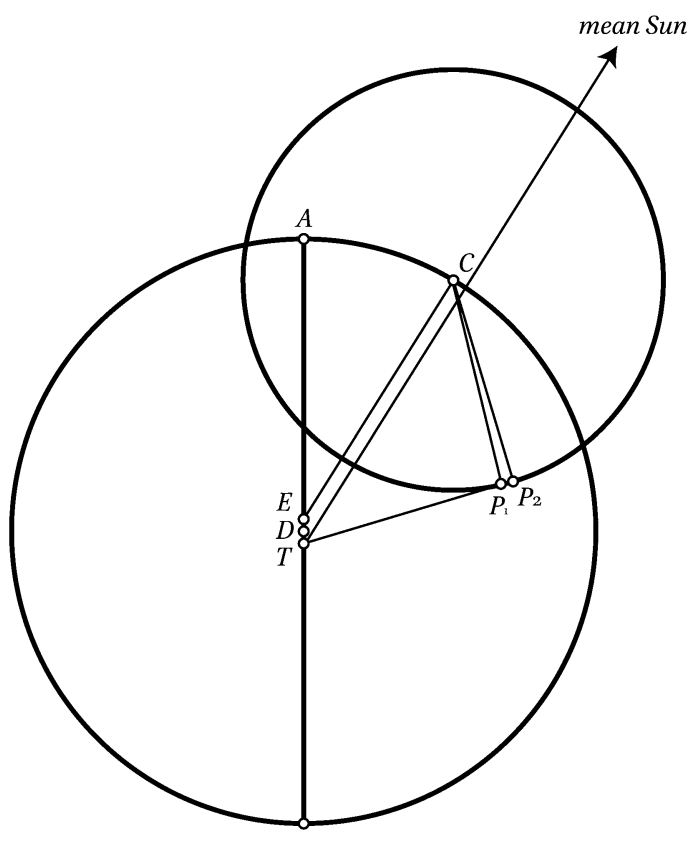

FIGURE 10.3 Ptolemy's equant model for Venus

point $P_{2}$ where a tangent from the Earth's center touches the epicycle (which we will call the "true tangent point"); however, in his demonstrations, Ptolemy consistently treats the planet as if it was at the true tangent point.

The difference in time between the moments when the planet is at greatest elongation and at the true tangent point varies more or less sinusoidally as a function of the mean argument of zodiacal anomaly (i.e. the elongation of the mean longitude of the epicycle's center from the apogee of the deferent, $A$ ), with maximum time difference at apogee and perigee and zero near quadrature. In the case of Mercury the maximum difference is less than a day, but for Venus it is as much as six days. Over that interval, Venus's elongation from the mean Sun can change by as much as 6 minutes, which is at the threshold of being significant. However, the only parts of Ptolemy's deduction of Venus's model that could potentially be affected by his conflation of the two configurations are the measurements of the deferent's eccentricity and the size of the epicycle, which are based on elongations found when the epicycle's center is close to either end of the apsidal line so that the discrepancy is near its maximum; and even here, the effects of the discrepancies at the apogee and perigee tend to cancel each other out with respect to the eccentricity. The determination of the apsidal line itself depends only on symmetries of greatest elongations, which are not affected by the conflation, while the deduction of 
the equant uses greatest elongations in which the mean argument of zodiacal anomaly is near $\pm 90^{\circ}$, so that the discrepancy is negligible.

The important point here is not the - at worst barely detectable-errors that Ptolemy introduces in his theory of the inferior planets by conflating the two configurations, but rather that the observations that he actually needs are of the planet at its true tangent points, and greatest elongations are merely an acceptable approximation that can be determined by comparison of observed longitudes of the planet with computed mean longitudes of the Sun. Further, he is not interested perse in the dates when the planet is at these points. Ideally, he would want to be able to observe the points themselves on any given date, and it is an inconvenience for him that the planet renders them vicariously visible only at scattered dates that have no natural correlation with the symmetries of the model that his analytical approach for the inferior planets seeks to exploit. ${ }^{17}$

How does one observe a greatest elongation? The arc of the planet's elongation from the mean Sun is of course not directly observable. What is observable is a criterion equivalent to the attainment of maximum elongation, namely the moment when the planet's true rate of longitudinal motion exactly equals the Sun's mean rate of longitudinal motion. Even this criterion could not be tested directly right at the sought date since the daily change in the daily longitudinal progress of Mercury and Venus around their greatest elongations is respectively on the order of four minutes and half a minute, in other words below the precision of the stellar coordinates on which ancient measurements of planetary longitudes depended (ten minutes in Ptolemy's star catalogue), to say nothing of the effects of measurement error. As was the case with solstices, one would have to estimate the moment of greatest elongation as the midpoint between two observed longitudes separated by several days, such that the increase in longitude is equal to the motion of the mean Sun in an equal interval. ${ }^{18}$ (Incidentally, all the reports of greatest elongations in the Almagest are ostensibly

17 As a corollary, it does not follow from the fact that the superior planets reach all elongations from the Sun that methods comparable to the ones he uses for the inferior planets were theoretically impossible for them. The counterpart of greatest elongations for the superior planets would be observations of the planet at the moment when it is travelling at its own mean rate of longitudinal motion. But aside from the-not insurmountableproblem of establishing the alignment of a superior planet's mean longitude, which is the counterpart of the mean Sun for the inferior planets, the smaller daily changes in longitude would have been an obstacle to accurate determination of these extremal moments. Swerdlow (1989:35) remarks on the impossibility of detecting the date of greatest elongation from the planet's day-to-day increase in longitude, but does not consider the magnifying effect of comparing longitudes separated by longer intervals. 
observations made while the planet was visible in the evening or morning, not statements of an interpolated "exact" time that could fall at any stage of day or night.)

Before considering the historical observations of greatest elongations in the Almagest, it will be helpful to look at four recent ones that Ptolemy tells us were among the observation reports that he personally received from Theon the Mathematician. For each, we give the report itself followed by the proleptic Julian calendar equivalent of Ptolemy's Egyptian calendar date, recomputation of what should have been observed according to modern theory, the planet's rate of longitudinal motion, and the approximate date when the planet was actually progressing at the rate of the mean Sun.

(1) "In the fourteenth year of Hadrian, Mesore 18 in the Egyptian calendar, in the evening, as we found in the observations we got from Theon, he says that (Mercury) was at its greatest distance from the Sun, $35 / 6^{\circ}$ behind the star on the heart of Leo ( $\alpha$ Leo)."19 (Almagest 9.9, H2.275)
Date of observation: AD 130, July 4, evening
Longitude of Mercury (17:30 UT): $127^{\circ} 24^{\prime}$
Elongation from Regulus: $3^{\circ} 28^{\prime}$
Rate of Mercury's longitudinal motion: $57^{\prime}$ / day
Greatest elongation: between July 3 and 4 (error $<1$ day)

(2) "Among the observations given to us by the mathematician Theon, we found one recorded in the sixteenth year of Hadrian, on Pharmouthi 21/22 in the Egyptian calendar, at which, he says, the planet Venus was at its greatest elongation as evening star from the Sun, and was the length of the Pleiades ${ }^{20}$ in advance of the middle of the Pleiades; and it seemed to be passing it a little to the south." (Almagest 10.1, H2.296)

Date of observation: AD 132, March 8, evening

Longitude of Venus (18:00 UT): $32^{\circ} 12^{\prime}$

Elongation from center of Pleiades: $1^{\circ} 41^{\prime}$

Rate of Venus's longitudinal motion: $50^{\prime}$ / day

Greatest elongation: February 21 (error 16 days)

\footnotetext{
19 The identifications of the fixed stars in the reports follow Toomer.

20 The apparent diameter of the Pleiades is slightly less than $2^{\circ}$; Ptolemy takes it to be $1^{\circ} 30^{\prime}$ (H2.296).
} 
(3) "Similarly, in the (observations we got) from Theon, we found that in the twelfth year of Hadrian, Athyr 21/22 in the Egyptian calendar, Venus as morning star had its greatest elongation from the Sun when it was to the rear of the star on the tip of the southern wing of Virgo ( $\beta$ Vir) by the length of the Pleiades, or less than that amount by its own diameter; and it seemed to be passing the star one moon to the north." (Almagest 10.1, H2.297-298)

Date of observation: AD 127, October 12, morning

Longitude of Venus (3:00 UT): $151^{\circ} 45^{\prime}$

Elongation from $\beta$ Vir: $1^{\circ} 2^{\prime}$

Rate of Venus's longitudinal motion: $1^{\circ} 6^{\prime}$ / day

Greatest elongation: September 22 (error 20 days)

(4) "In the (observations) given to us by Theon we find that in the thirteenth year of Hadrian, Epiphi 2/3 in the Egyptian calendar, Venus was at its greatest elongation from the Sun as morning star, and was $12 / 5^{\circ}$ in advance of the straight line through the foremost of the three stars in the head of Aries ( $\gamma$ Ari) and the star on the hind leg ( $\mu$ Cet), while its distance from the foremost star of those in the head was approximately double its distance from the star on the leg." (Almagest 10.2, H2.299)

\author{
Date of observation: AD 129, May 20, morning \\ Longitude of Venus (2:00 UT): $12^{\circ} \mathrm{o}^{\prime}$ \\ Elongation from line through $\mu$ Cet and $\gamma$ Ari: $1^{\circ} 29^{\prime}$ \\ Rate of Venus's longitudinal motion: $1^{\circ} 3^{\prime} /$ day \\ Greatest elongation: May 6 (error 14 days)
}

These reports present us with an alarming disjunction between their positional information, which is accurate by the standards of ancient ancient naked-eye observations, and the very large deviations of three of the dates from the actual dates of greatest elongation. The exception is observation (1), which is essentially a correct determination of the date and position of Mercury's greatest elongation. Ptolemy's wording explicitly credits Theon with the identification of the date as that of greatest elongation. But this is the case also with observation (2); and in this observation, as well as in (3) and (4), the error in the date of greatest elongation is so large that one has difficulty believing that it is merely due to defects in the observations or sloppy analysis.

Swerdlow argues persuasively that Ptolemy could not have originally obtained the structure and parameters of his model for Venus by the route that 
he sets out in Almagest 10, and, drawing attention to the fact that many of the ostensible greatest elongations that Ptolemy cites have dates significantly differing from the actual dates of the phenomenon, he offers the surely correct explanation that Ptolemy has chosen observations that merely bring the planet close to greatest elongation while situating the mean Sun almost exactly in configurations relative to the planet's apsidal line that permit the Almagest's didactically elegant deduction through symmetries. ${ }^{21}$ I think one can take this reconstruction a bit further.

As we saw above, a greatest elongation from the mean Sun is a configuration in which the longitude of an inferior planet approximately coincides with the longitude of the true tangent point of its epicycle on either the positive (evening) or negative (morning) side of the epicycle's center, so that one can obtain from observation and calculation the elongation of the true tangent point from the mean Sun. If we consider the elongations of the evening and morning true tangent points from the mean Sun as two functions of the longitude of the mean Sun, Ptolemy's planetary models predict that the evening and morning functions will be exact mirror images of each other with respect to the apsidal line. The compromises that Swerdlow has identified were necessitated by the fact that the greatest elongations available to Ptolemy from his observational records would not have included a set falling in locations that allowed him to exploit this symmetry in the most direct manner.

In Almagest 10.1 Ptolemy professes to find Venus's apsidal line as the line of symmetry of two pairs of equal but opposite greatest elongations; the demonstration imposes no constraint on the specific magnitudes of the elongations so long as they are pairwise equal and opposite, and no constraint on the corresponding locations of the mean Sun so long as both pairs are symmetrically placed with respect to the same apsidal line. Then in 10.2 he finds the deferent's eccentricity and the epicycle's radius using a pair of greatest elongations with the mean Sun at opposite ends of the apsidal line; here the magnitudes as well as the positions of the mean Sun are critical. In each of the three pairs of greatest elongations, one is by Theon and the other by Ptolemy. It is noteworthy that all four of the observations used in 10.1 as well as the earlier of those used in 10.2 (the Theon observation from AD 129) are on dates later than the true greatest elongations by more or less consistent intervals in the range $16 \pm 4$ days, while the remaining observation in 10.2 (an observation by Ptolemy from $\mathrm{AD} 136, \mathrm{H} 2.300$ ) is 25 days before a true greatest elongation that is also the one falling closest to one of the observations used in 10.1.

21 Swerdlow (1989). 
What I suspect to have happened is that Ptolemy started off with a set of observations of Venus at, or very close to, the true dates of greatest elongation, partly drawn from Theon's observations and partly from his own. Theon may himself have identified certain observations as greatest elongations, as Ptolemy attests that he did for the Mercury observation cited in Almagest 9.9. Whoever did it, the method would have been the one outlined above, taking an observation from the halfway date of two fairly widely spaced observations between which Venus's progress was approximately equal to that of the mean Sun. Either these did not yield pairs exhibiting the exact symmetries needed for the demonstration of the apsidal line, or Ptolemy had foreknowledge of the apsidal line that he was seeking, or both; in any case he chose to apply a roughly consistent temporal shift to two pairs of greatest elongations that nearly provided the symmetries, as well as to one more that appeared to have the mean Sun near one end of the apsidal line. For each of these new, symmetrically paired dates, he selected one genuine observation among Theon's that was not indicated by Theon as a greatest elongation but that fell as close as possible to the earlier date. (In other words, I disbelieve Ptolemy's claim that Theon called the observation of AD 132, March 8 a greatest elongation.) Then he fabricated - not interpolated - an observation of his own that supplied the exact equal-but-opposite counterpart near the later date. Again, for the eccentricity and epicycle radius he selected a genuine Theonic observation, not of a greatest elongation, close to the shifted date of the apsidal greatest elongation, and fabricated an observation of his own with the mean Sun in the diametrically opposite position, and with an elongation chosen so as to lead to values for the eccentricity and epicycle radius that Ptolemy had decided on in advance.

Given a series of daily, or near-daily, observations of an inferior planet's position over a sufficient time span around the estimated date of greatest elongation Ptolemy, or Theon, should have been able to establish which observation was closest to the moment of greatest elongation subject to errors of one or two days. A span of as much as twenty days was desirable for Venus; for the rapidly accelerating Mercury just eight or ten days should have sufficed, while beyond about twelve days the asymmetry of the elongation function around its extreme becomes too pronounced for the method of taking the midway date to work. The large discrepancies in the reports of Venus from Almagest 10.12 , since they can be accounted for by the systematic shift conjectured above, are not good evidence that this level of accuracy was not attained in practice. Ptolemy's remaining two greatest elongations of Venus, used in Almagest 10.3 to locate the equant, are respectively just three days and one day off from the correct dates, and even these discrepancies are in part due to the circumstance 
that his procedure requires two observations with the mean Sun near exact quadrature relative to the apsidal line. The discrepancies for his recent reports of Mercury's greatest elongations, averaging under three days with a maximum discrepancy of six days, can also be explained as adjustments for the sake of his deductive arguments.

Ptolemy's historical reports of greatest elongations all appear in a single chapter, Almagest 9.7, the counterpart for Mercury of Almagest 10.1 for Venus in which the planet's apsidal line is determined as the line of symmetry of two pairs of equal but opposite recent observations of greatest elongations. Only for Mercury, however, does Ptolemy carry out a similar determination using a set of historical observations, whereas in the case of Venus he writes that "the available ancient observations did not supply us with exact pairs of positions for this purpose" (Almagest 10.1, H2.296).

In introducing the historical observations in 9.7, he characterizes them as "the ancient observations made near the greatest elongations" (H2.264) and none of these reports has wording comparable to that of the two reports from Theon discussed above asserting that the observers had identified the dates as falling on or close to greatest elongations. The implication appears to be that Ptolemy, or some intermediary such as Hipparchos, selected the cited observations from a sufficiently abundant transmitted corpus so that it was possible to find an observation of Mercury approximately on the halfway date of two chronologically proximate observations between which the planet's average speed was close to that of the mean Sun.

Two of the observations are Babylonian:

In the $75^{\text {th }}$ year according to the Chaldeans, Dios 14, at dawn, (Mercury) was half a cubit above the southern scale ( $\alpha$ Lib).

$\mathrm{H} 2.267$

In the 67th year according to the Chaldeans, Apellaios 5 , at dawn, (Mercury) was half a cubit above the northern forehead of the scorpion ( $\beta$ Sco). H2.268

Like the one other Babylonian planetary observation in the Almagest (in 11.7, $\mathrm{H} 2.419$ ), these appear to be faithful translations of records of the type known as "normal-star passages" which occur frequently in the Babylonian Astrono- 
mial Diaries, Goal Year Texts, and Excerpt Texts; ${ }^{22}$ the year count is according to the Seleucid Era, the calendar date is probably a direct rendering of the original Babylonian calendar date with schematic substitution of a Macedonian month name, and the metrology of cubits and fingers literally reproduces the meaning of the Babylonian units. ${ }^{23}$ Ptolemy interprets the reports as meaning that the planet was due north of the named fixed star in ecliptic coordinates, i.e. at the same longitude as the star, on the date of the observation. His procedure, therefore, is to express the date in his own chronological system (Egyptian calendar, Era Nabonassar), equate the planet's longitude with the longitude of the star provided by his own star catalogue, corrected for precession, and calculate the mean Sun for the date using his own solar theory.

The survival of a significant fraction of the cuneiform tablets of the Babylon astronomical archive makes it possible for us to perceive several obstacles to using planetary observations from the archive in the way that Ptolemy professes to be doing — obstacles that would only have become more severe if the intermediate stages of their transmission involved losses and distortions in the observational record. In the first place, observations of Mercury at a particular time of night (i.e. shortly after sunset or shortly before sunrise) were not available in all ranges of longitude. In Almagest 13.8 (H2.597-598) Ptolemy states that the planet cannot make an appearance in the evening when it is around the beginning of Scorpio or in the morning when around the beginning of Taurus; and the Babylonian mathematical models for calculating visibility dates for Mercury similarly assume that intervals of evening visibility will be "missed" when the predicted first visibility would occur in Libra or the first few degrees of Scorpio, while morning visibility will be "missed" for predicted first visibility occurring in much of Aries and Taurus. ${ }^{24}$ But the conditions for simply sighting the planet were more generous than those allowing its position to be determined, since that required at least one identifiable star to be visible together with the planet. The Babylonian observational records show that the longitudinal ranges within which Mercury was never or rarely observable together with a Normal Star in the morning or evening were much more extensive than the ranges in which visibility was "missed"; in fact for either time of night observations were practically limited to about half the ecliptic, with further gaps caused by the highly uneven distribution of the Normal Stars

\footnotetext{
22 Jones (2004).

23 Jones (2006: 273-276); Stern (2012: 238-243).

24 Neugebauer (1975:1.403-404).
} 


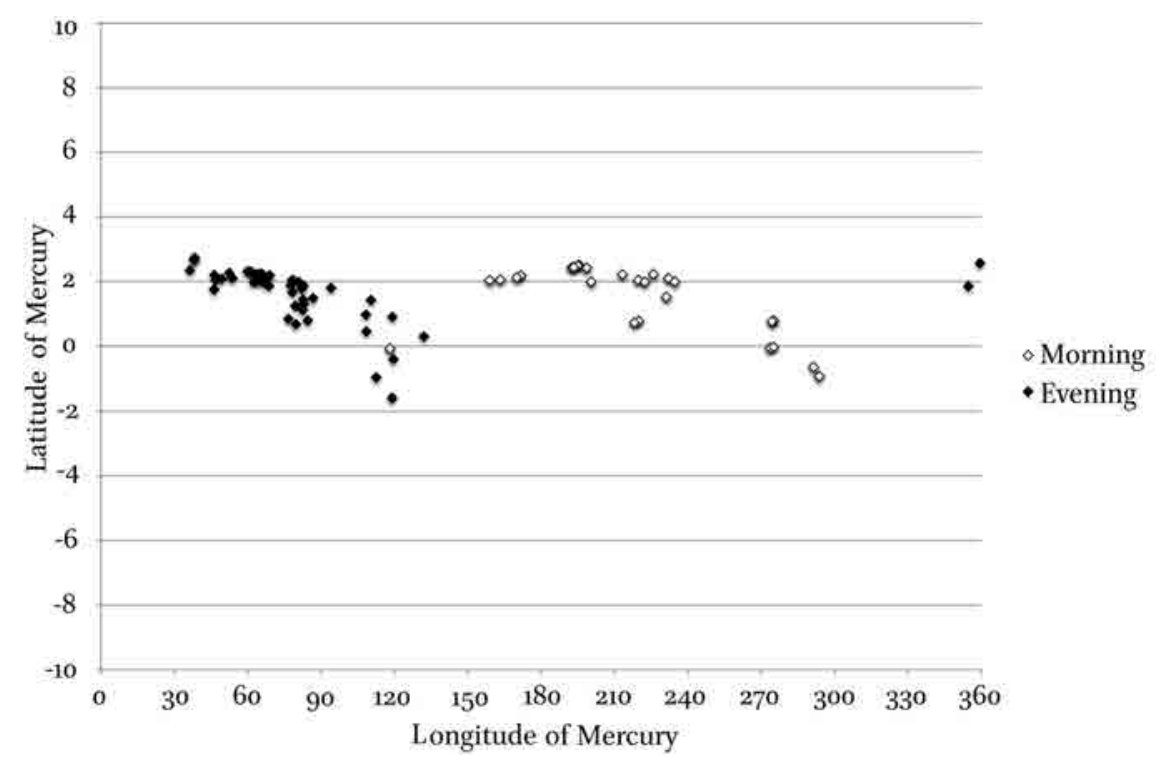

FIGURE 10.4 Locations of Mercury on the dates of surviving Babylonian Normal Star observations

(Fig. 10.4). ${ }^{25}$ The major "blacked-out" ranges approximately coincide with Aquarius through Cancer for morning observations and Virgo through Aquarius for evening observations; the corresponding "blacked-out" ranges of longitude of the mean Sun are roughly Pisces through Cancer for morning observations and Leo through Aquarius for evening observations (Fig. 10.5). These gaps are all the more deleterious because Mercury's geocentric apsidal line falls roughly in their middles, thus greatly limiting the opportunities of finding symmetrically placed observations of equal but opposite greatest elongations. As Swerdlow has remarked, this was probably a major factor contributing to Ptolemy's erroneous placement of Mercury's apogee at much too low a longitude, near the beginning of Libra. ${ }^{26}$

Moreover, the chronological spacing of the Babylonian observations was not dense. At most one observation was recorded for each Normal Star that a planet passed, usually on the date when the planet came closest in longitude to the star. Only in a few regions of the ecliptic were Normal Stars closely enough spaced to give opportunities for several Normal Star passages to be

25 Figs. 4-7 were prepared from the database of Babylonian planetary Normal Star observations described in Jones (2004:479-481).

26 Swerdlow (1989:46-47). 


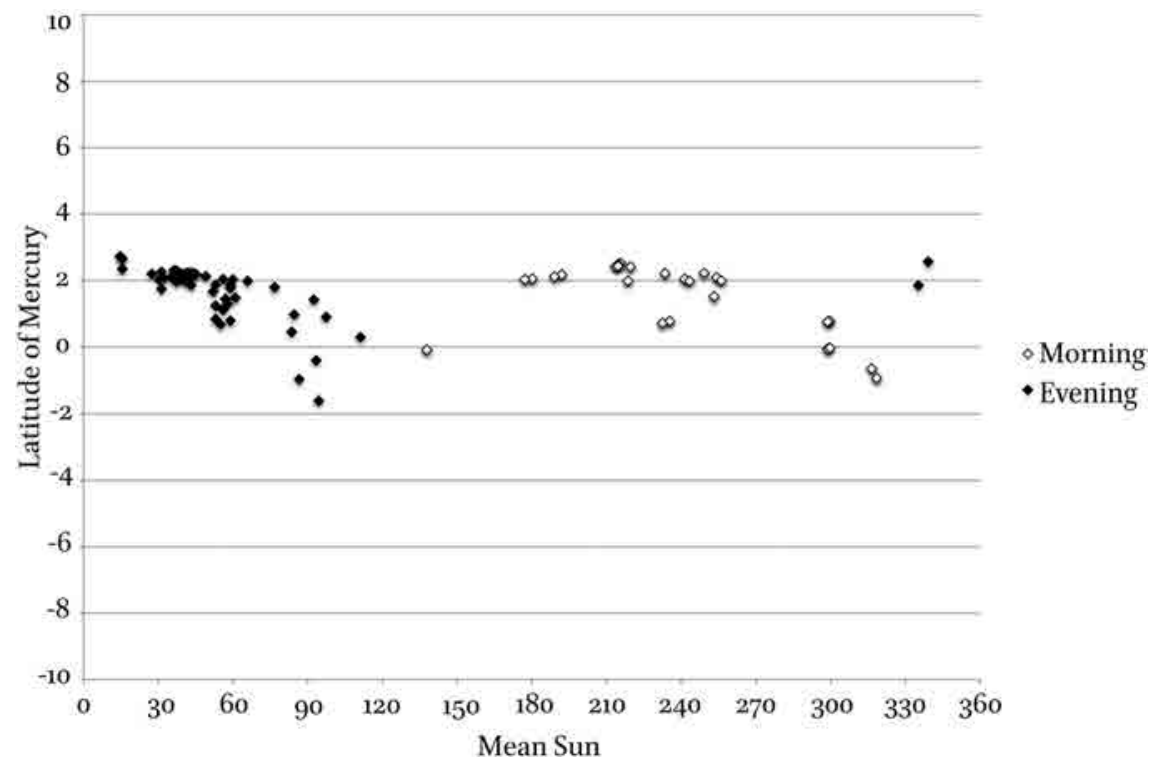

FIGURE 10.5 Locations of mean Sun on the dates of surviving Babylonian Normal Star observations of Mercury

observed in rapid succession. In the case of Mercury, during the comparatively brief periods of the planet's visibility, it was seldom that the Babylonian observers recorded more than two Normal Star passages. Hence the conditions would rarely have been satisfied for determining the approximate date of a greatest elongation, let alone for finding an actual observation made on that date. How Ptolemy satisfied himself that the two Babylonian reports cited in Almagest 9.7 were close to dates of greatest elongation is something of a mystery.

These are limitations that would have been obvious to Ptolemy simply from perusing the transmitted records. It would not have been quite as straightforward a matter for him to know what the relationship was between a report of the kind he cites, stating that a planet was so many units above or below a Normal Star, and the actual configuration of the planet and star. He assumes that the absence of a reported distance east or west (or as the Babylonian records would have said, "behind" or "in front") means that the planet was longitudinally aligned with the star. With the resources of modern theory at our disposal, we know that this was by no means the case. Most of the planetary Normal Star observations have no indication of east-west separation, despite the fact that observations were made at about the same time on successive days. Taking in aggregate all the reports of a particular planet recorded as being simply above or below any Normal Star, the relative positions of planet relative to star, 


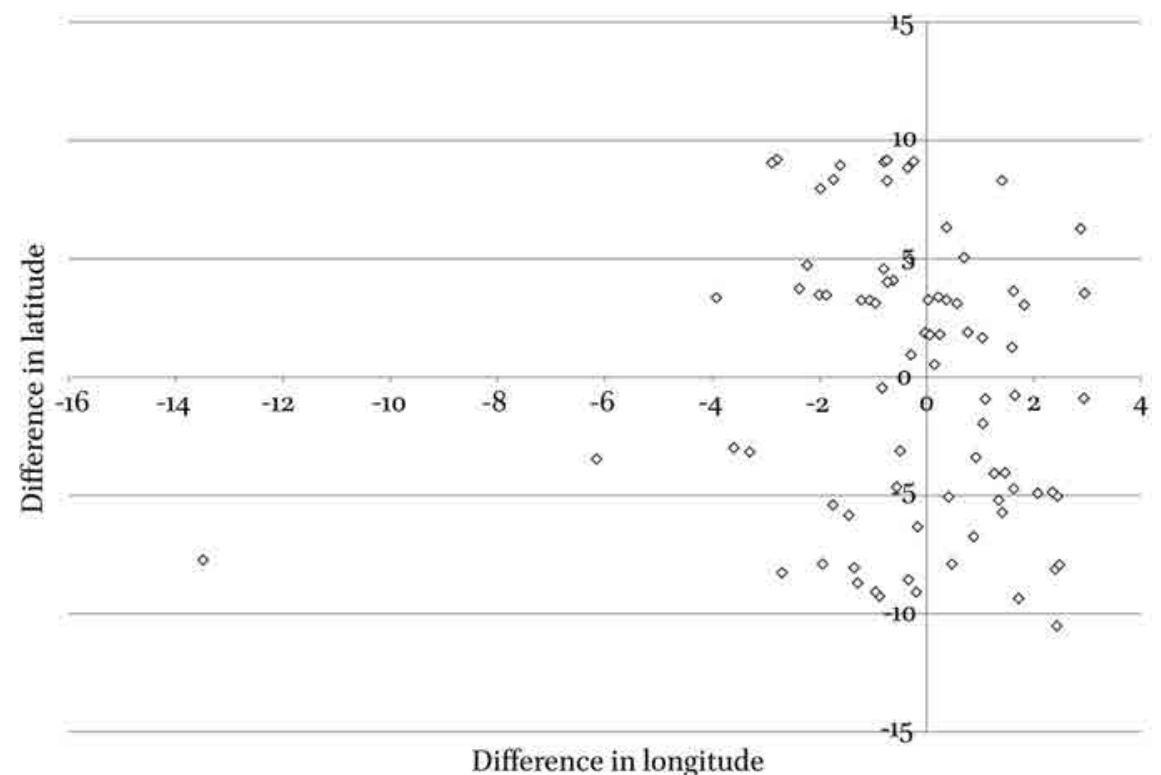

FIGURE 10.6 Locations of Mercury relative to Normal Star on dates of surviving Babylonian observations

as reconstructed by modern theory, form a cloud that, in the case of an inferior planet, has substantial longitudinal spread (Figs. 10.6-7). ${ }^{27}$ If Ptolemy had access to a large enough number of the Babylonian observations, he ought to have been able to infer this aspect of observational practice simply from the abundance of reports lacking east-west distance specifications.

On top of all these problems, he must have been conscious that the translation of Babylonian calendar dates into his Egyptian calendar framework was subject to uncertainties of \pm 1 day since the beginnings of Babylonian months were determined by observation or calculation of lunar crescent visibility, not a schematic pattern that could be reliably mapped on to another calendrical framework. An error of as little as a day would have grave consequences for any analysis Ptolemy based on an observation, because it would cause an error of almost a degree in the assumed longitude of the mean Sun. In short, the Babylonian corpus was a highly unsatisfactory resource for obtaining greatest elongations.

27 Some of the spread in Figs. 6-7 is due to uncertainties in converting Babylonian calendar dates to the proleptic Julian calendar. 


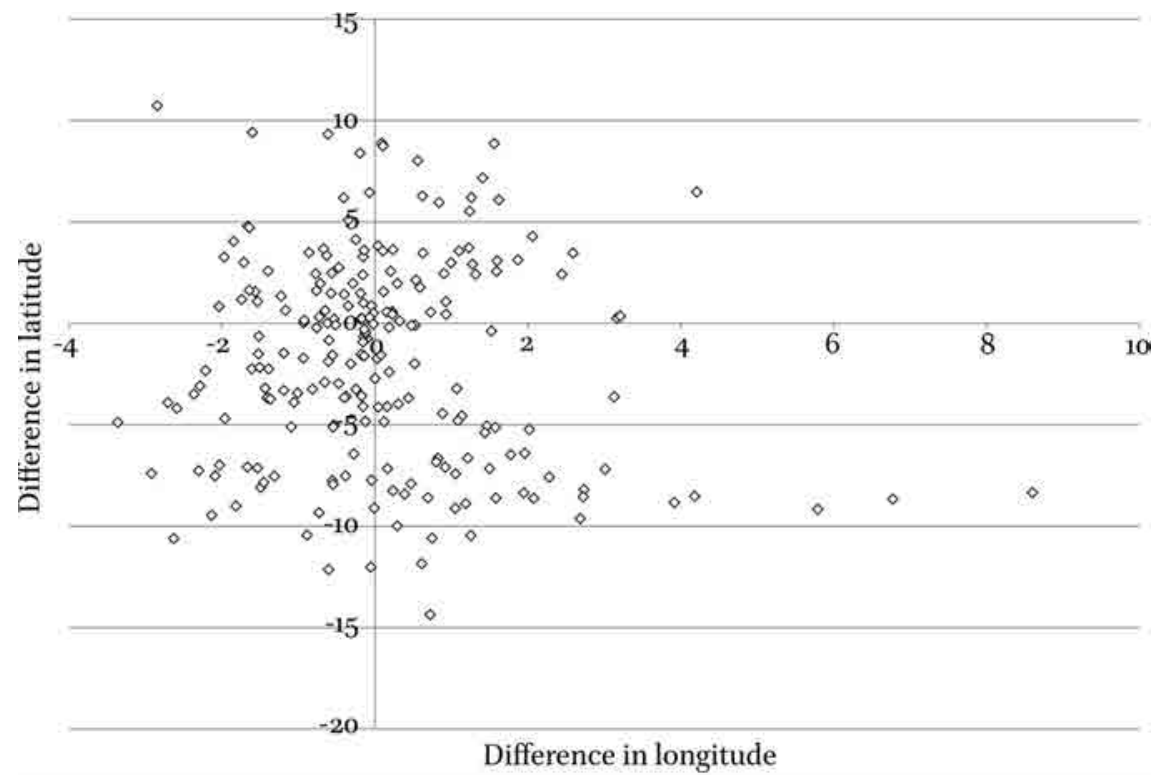

FIGURE 10.7 Locations of Venus relative to Normal Star on dates of surviving Babylonian observations

We lack an independent source of information about the Dionysian observations that supplied Ptolemy with his remaining four historical greatest elongations. Nevertheless the reports that he provides allow us to notice some differences in the observational practices underlying this corpus compared with the Babylon archive. Two specimens will illustrate the character of these reports; the first of them is one of his four greatest elongations in 9.7, while the second is an observation cited not as a greatest elongation but as an observed position used to correct the planet's periodicities in 9.10:

In the 23rd year according to Dionysios, Hydron 21, at dawn, Stilbon (Mercury) was 3 Moons to the north of the brightest star in the tail of the goat-fish.

$\mathrm{H} 2.264$

In the 21st year according to Dionysios ... Skorpion 22, ... at dawn, Stilbon was 1 Moon to the rear of the straight line through the northern forehead of the scorpion and the middle one, and was 2 Moons to the north of the northern forehead ... Furthermore it is clear that it had not yet reached its greatest elongation as morning star, since 4 days later, on Skorpion 26, it is recorded that its distance from the same straight line towards the rear 
was 11/2 Moons; for the elongation had become greater, the Sun having moved about 4 degrees, but the planet half a Moon.

H2.288-289

Aside from using a different calendrical system and different metrology, the first of these examples resembles the Babylonian observations in reporting the planet's position as simply north of a reference star, and Ptolemy interprets it in the same way, as implying that the planet and star had the same longitude. ${ }^{28}$ In contrast to the situation with respect to the Babylonian observations, here it seems that Ptolemy's interpretation is legitimate. The second example seems to be more typical of the records for Mercury from this corpus, since two of the other reports in 9.7 are of the same kind: the planet's location is specified in east-west distance relative to an imagined "straight line" through a pair of stars as well as in north-south or radial distance relative to a single star, thus providing a pair of coordinates fixing the planet's position. (The fourth report in 9.7 is given only in Hipparchos's reduction to an elongation in degrees from a star, so the original form cannot be recovered.) In the second example quoted above, Ptolemy also informs us that another observation was recorded four days later, allowing him to confirm that the planet had not yet reached its greatest elongation. Hence we can see that this corpus potentially contained a density of observations of Mercury that would have enabled him to estimate some dates of greatest elongation and to find observed positions on these dates.

Were the Dionysian observations of Mercury subject to the large blackout zones that we found in the Babylonian records? The more southerly latitude of their presumed place of observation, Alexandria, ought to have offered slightly more favorable conditions for sighting Mercury together with nearby stars. As it happens, all the reports that Ptolemy quotes in what looks like their original wording had Mercury within the zones where the Babylonian records attest to favorable conditions of visibility. However, the last of the reports in Almagest 9.7, which Ptolemy gave only according to Hipparchos's reduction, had Mercury visible in the evening about two-thirds of the way through Virgo, more than $30^{\circ}$ inside the Babylonian evening blackout zone. ${ }^{29}$

28 Ptolemy's single historical observations of Mars and Jupiter from this corpus, in Almagest 10.9 and 11.3, are also ostensible alignments of planet with star, indeed worded as if they were seen as occultations.

29 One might expect Ptolemy's reports of his own observations of Mercury at Alexandria to provide further evidence for the extent of the blackout zones at this terrestrial latitude. 
As was the case with the Babylonian reports, the calendar conversions may have been subject to uncertainty, though for a different reason. The calendar of Dionysios was a solar calendar, apparently invented purely for astronomical applications, with months named after the zodiacal signs through which the Sun passes in the course of the year. If, as seems likely, the calendar likely followed a cycle of three 365 -day years followed by a 366 -day year, the mapping of its dates on to Ptolemy's chronological system ought to have been unambiguous. However, some of the Dionysian planetary observations cited in the Almagest describe configurations that were in effect as much as two days before or after the Egyptian calendar dates that Ptolemy assigns to them..$^{30}$ A plausible explanation of these discrepancies is that Ptolemy, or a predecessor who carried out the date conversions, lacked complete information about how the Dionysian year was partitioned into its zodiacal months.

On the whole, the Dionysian reports were far more suitable for extracting greatest elongations than the Babylonian corpus, and this should have been obvious to Ptolemy. But there was a serious problem with them that becomes apparent if we consider the entire selection of historical observations in Almagest 9.7. Unlike Venus, Mercury's synodic period is short and does not have near resonance with the solar year like the one that causes Venus to repeat its phenomena at nearly the same longitudes after only five synodic cycles. Hence it does not require a long span of time to accumulate a reasonably dense and even distribution of greatest elongations of both kinds throughout the ecliptic; in just thirteen years, there will be 41 evening and 41 morning greatest elongations corresponding to positions of the mean Sun spaced an average of under $9^{\circ}$ apart (Fig. 10.8). Some of these would have fallen within the "blackedout" zones, but in other parts of the ecliptic the observational practices of the Dionysian observers ought to have provided Ptolemy with sufficient observations to provide him with historical greatest elongations close to most of the situations that he wanted.

But this was clearly not the case: he tells us that he was unable to find suitable pairs of equal but opposite greatest elongations, so instead he twice constructs pairs by matching a single Dionysian observation with a simulated equal but opposite greatest elongation linearly interpolated between two actual observa-

Their relevance is uncertain however, both because he professes to have observed them using his armillary instrument and because suspicion of tampering generally adheres to his reports of his own observations. A couple of his reports had Mercury visible in the morning very deep within the Babylonian blackout zone, at about Taurus $20^{\circ}$ (Almagest 9.7, $\mathrm{H} 2.262-263)$ and Gemini $20^{\circ}$ (9.9, H2.275). 


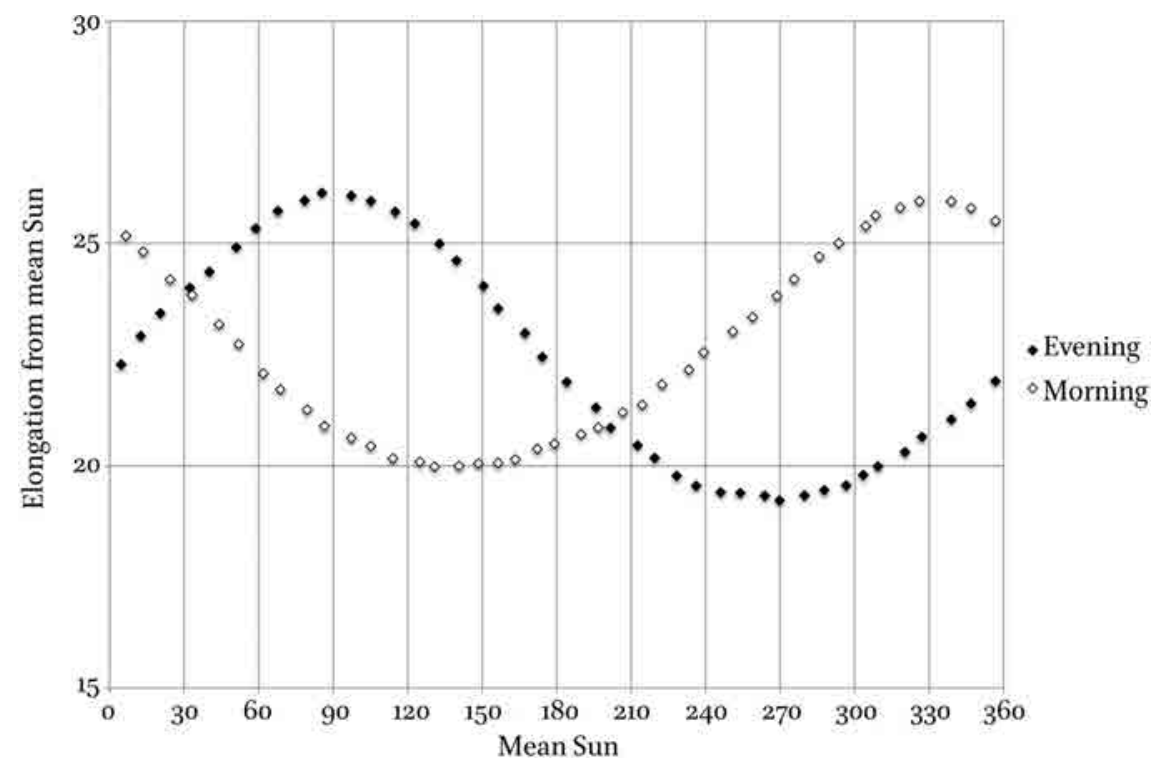

FIGURE 10.8 Greatest elongations of Mercury from mean Sun computed by modern theory for $270-258 B C$

tions for which the difference in the mean Sun is in one instance $331 / 3^{\circ}$ and in the other $192 / 3^{\circ}{ }^{31}$ What is more, the second time that he does this, he uses the two Babylonian observations that we have referred to above instead of the far preferable Dionysian observations to obtain his simulated matching observation. The reason that he was compelled to resort to these measures was almost certainly that he did not have many years' worth of Dionysian observations, I would suppose not much more than eight years from 265 through $257 \mathrm{BC}$ from which all the examples cited in the Almagest come.

The Dionysian observers, whoever they were, were active from at least as early as $272 \mathrm{BC}$ (the year of Ptolemy's one historical observation of Mars in Almagest 10.9, $\mathrm{H2.352}$ ) to at least as late as $241 \mathrm{BC}$ (the year of the historical observation of Jupiter in Almagest 11.3, H2.386, and the observation of the same planet in POxy astron. 4133). The two observation reports for Jupiter (Almagest 11.3, H2.386 and POxy astron. 4133) that give us the terminus post quem for the end of their observational program apparently are known to us only because an astronomer active in AD 104/105 used them to investigate a 344-year period

31 Applying linear interpolation was not unreasonable in these situations, since the greatest elongations in question were in parts of the ecliptic where the elongation varies fairly linearly as a function of the longitude of the mean Sun. 
relation for Jupiter. ${ }^{32}$ Similarly, the report for Mars (Almagest 10.9, H2.352) that gives us the terminus ante quem for the program's beginning is likely to have survived and reached Ptolemy's hands by way of an astronomer active around AD 92-Agrippa or Menelaos? — who was investigating a 363-year period relation for Mars. The Dionysian observers' records probably took in all the planets throughout the several decades of their work. What is not at all plausible is that they concentrated all their attention on Mars for just a short interval around 272, on Mercury for a few years around 260, and on Jupiter around 241. Ptolemy's small chronological window on the Dionysian Mercury observations is further confirmation that he depended for his historical observations on intermediate sources that transmitted only a small selection from the original corpora.

\section{$7 \quad$ Conclusion}

It is a commonplace to say that, because he lived in Alexandria, Ptolemy had access to uniquely rich resources of past astronomy through the library of Alexandria. ${ }^{33}$ But the bulk and richness of the Alexandrian library's holdings have been greatly exaggerated even for the Hellenistic period, and when it comes to Ptolemy's time we have practically no reliable information about the library's condition. ${ }^{34}$ In fact thanks to Galen we know much more about libraries in Antonine Rome than in contemporary Alexandria, and it would probably be safest to suppose that conditions in Alexandria were similar tobut perhaps not quite as good as - those in the capital, with a multiplicity of smaller public and private collections rather than a single predominating repository imagined anachronistically as a precursor of the modern research library. ${ }^{35}$ Ptolemy is not known to have had any status that would have given him special access to out-of-the-way archives. It would always have been easier to find and consult the recent astronomical literature than works produced in or before the early Hellenistic period.

The solstices, equinoxes, greatest elongations, and oppositions on which Ptolemy's solar and planetary theories so heavily depended represented a dis-

$32 \quad$ Jones (1999).

33 E.g. Pedersen (1974:13): "It was Ptolemy's personal merit that he was more careful than most other Hellenistic authors in quoting his sources and acknowledging his predecessors; but it was the great Alexandrian library which enabled him to do so."

34 Bagnall (2002).

35 Nicholls (2011). 
tillation of large sets of direct observations, and his limited and indirect access to historical observation records rendered them a problematic source for such interpolated observations. On the one hand, he had a few-very few-solstice reports going back as far as the fifth century ВС, but with these the process of determining a solstice date from multiple individual observations, whatever form it took, had been carried out at the time, so that Ptolemy could only make a rather pessimistic guess about their quality. On the other hand, he had no historical observations of greatest elongations of the inferior planets reported as such, and probably also no historical reports of oppositions of superior planets with the mean Sun, but only reports of observed positions of the planets relative to fixed stars.

It should have been possible for Ptolemy to obtain some reasonably accurate interpolated dates and positions of mean oppositions; these would have required only a pair of observed positions close to the event, or even just a single observation if it fell close enough so that one could use an estimate of the planet's rate of retrograde motion. Hence he could have tried to establish an apsidal line and eccentricity for a superior planet from historical observations using the same method of analysis that he applies in the Almagest to recent observations, though the results would likely not have been sensitive enough to demonstrate more than the weak conclusion that the phenomena "fit" the assumption of a sidereally fixed apsidal line. The difficulty with which Ptolemy cobbles together a not entirely satisfactory set of historical greatest elongations of Mercury to furnish the Almagest's specious demonstration that its apsidal line is sidereally fixed shows that the historical observations available to him could not have provided an adequate supply of greatest elongations of the inferior planets for purposes of research.

\section{Bibliography}

Bagnall, R.S., 2002, "Alexandria: Library of Dreams", Proceedings of the American Philosophical Society $146,348-362$.

Dessau, H., 1904, “Zu den milesischen Kalendarfragmenten”, Sitzungsberichte der Königlich-Preussischen Akademie der Wissenschaften, Phil.-hist. Kl. 23, 266-268.

Diels, H. and Rehm, A., 1904, "Parapegmenfragmente aus Milet", Sitzungsberichte der Königlich-Preussischen Akademie der Wissenschaften, Phil.-hist. Kl. 23, 92-111.

Fournet, J.-L. and Tihon, A., 2014, Conformément aux observations d'Hipparque: le Papyrus Fouad inv. 267 A (Louvain-la-neuve: Université catholique de Louvain).

Jones, A., 1999, "A Likely Source of an Observation Report in Ptolemy's Almagest", Archive for History of Exact Sciences 54, 255-258. 
Jones, A., 2004, "A Study of Babylonian Observations of Planets Near Normal Stars", Archive for History of Exact Sciences 58, 475-536.

Jones, A., 2005, "In order that we should not ourselves appear to be adjusting our estimates ... to make them fit some predetermined amount", in J.Z. Buchwald and A. Franklin (ed.), Wrong for the Right Reasons (Dordrecht: Springer), 17-39.

Jones, A., 2006, "Ptolemy's Ancient Planetary Observations", Annals of Science 63, 255290.

Jones, A., forthcoming, "The Miletos Inscription on Calendrical Cycles: IMilet inv. 84 + inv. 1604."

Lehoux, D., 2005, “The Parapegma Fragments from Miletus”, Zeitschrift für Papyrologie und Epigraphik 152, 125-140.

Neugebauer, O., 1975, A History of Ancient Mathematical Astronomy (New York: Springer).

Nicholls, M.C., 2011, "Galen and Libraries in the Peri Alupias", Journal of Roman Studies 101, 123-142.

Ossendrijver, M., 2012, Babylonian Mathematical Astronomy:Procedure Texts (New York: Springer).

Pedersen, O., 1974, A Survey of the Almagest (Odense: Odense University Press).

Rawlins, D., 1990, "Hipparchos' ultimate solar orbit \& the Babylonian tropical year", Dio 1.1, 49-66.

Stern, S., 2012, Calendars in Antiquity: Empires, States, and Societies (Oxford: Oxford University Press).

Swerdlow, N.M., 1989, "Ptolemy's Theory of the Inferior Planets", Journal for the History of Astronomy 20, 29-60.

Swerdlow, N.M., 2004, "The Empirical Foundations of Ptolemy's Planetary Theory", Journal for the History of Astronomy 35, 249-271.

Toomer, G.J., 1984, Ptolemy's Almagest (London: Duckworth). 